\title{
Impact de l'usage des gants médicaux sur l'observance de l'hygiène des mains au cours des soins au Centre Hospitalier et Universitaire du Point G de Bamako
}

\author{
Impact of medical gloves use on adherence to hand hygiene in health care at the \\ University Hospital of Point G Bamako \\ L.Bengaly ${ }^{1}$, S. Fomba ${ }^{2}$, M. Keita ${ }^{3}$, A T. Traoré ${ }^{1}$, Z. Harouna ${ }^{1}$, A. Fofana ${ }^{4}$, D. Traoré ${ }^{5}$, J P.Dembélé ${ }^{4}$
}

1- Service de Pharmacie Hospitalière (Centre Hospitalier et Universitaire du Point G).

2- Programme National de Lutte contre le Paludisme (PNLP).

3- Service Anesthésie-réanimation et Urgences (Centre Hospitalier et Universitaire du Point G).

4- Service de Maladies Infectieuses et Tropicales (Centre Hospitalier et Universitaire du Point G).

5- Service de Chirurgie B (Centre Hospitalier et Universitaire du Point G).

Auteur correspondant: Dr Loséni Bengaly CHU Gabriel Touré ; Email: losbengaly@yahoo.fr

\section{Résumé}

Objectif : Évaluer l'impact de l'usage des gants médicaux sur l'observance de l'hygiène des mains et promouvoir leur usage approprié.

Matériel et Méthodes: Il s'agissait d'une étude interventionnelle avec une évaluation avant et après intervention. Sa mise en œuvre a été réalisée entre avril 2010 et octobre 2011 au Centre Hospitalier et Universitaire du Point G. Le recueil du consentement éclairé et les observations ont été effectués auprès du personnel soignant ayant un contact direct avec les patients avant et après intervention. Ces observations ont été discrètes mais ouvertes, menées auprès des soignants préalablement informés du but et du programme des activités.

Résultats : Au total, 143 fiches de consentement éclairé ont été retournées sur une prévision de 274 fiches, soit $52,19 \%$. Les observations ont donné un taux d'observance globale de 52,05\% avant intervention contre $42,97 \%$ après intervention $(p=0,0017)$. Le taux global de port de gants était de $23,30 \%$ et $27,20 \%$ respectivement avant et après intervention $(\mathrm{p}=0,12)$.

Conclusion : Les résultats des deux évaluations avant et après intervention ont montré une amélioration du taux global de port des gants qui n'était pas significative mais sans impact sur l'observance de l'hygiène des mains.

Mots clés: Gants médicaux ; Hygiène des mains ; Mali ; Point G ; Soins.

\begin{abstract}
Goal: This research aimed to assess the impact of medical gloves use on adherence to hand hygiene and to promote their appropriate use.

Material and Methods: This was an interventional study with an evaluation before and after intervention. It was carried out from April 2010 to October 2011 at the University Hospital of Point G. The collection of informed consent and the observations were done before and after intervention among the health staff having direct contact with patients. These observations were discreet but opened, carried out among health staff previously informed of the purpose and the activitie's program.

Results: A total of 143 informed consent forms were returned for a forecast of at least 274 health staff that is to say $52.19 \%$.. During the observations we found an overall compliance of $52.05 \%$ before intervention and 42.97 $\%$ after intervention $(\mathrm{p}=0,0017)$. The global rate of gloves use was $23.33 \%$ and $27.20 \%$ respectively before and after intervention $(\mathrm{p}=0,12)$.

Conclusion: The results of both evaluations showed a no statistically significant improvement of the global rate of medical gloves use but without an impact on the compliance to hands hygiene.
\end{abstract}

Keywords: Medical gloves; Hands hygiene; Mali; Point G; Health care.

\section{Introduction}

Le Centre Hospitalier et Universitaire (CHU) du Point $\mathrm{G}$ a été sélectionné en 2007 comme site pilote de l'Organisation Mondiale de la Santé de la région africaine (OMS- AFRO) pour tester la mise en œuvre des recommandations OMS sur l'hygiène des mains à travers la stratégie multimodale OMS de l'amélioration de l'hygiène des mains au cours des soins de santé [i- 4]. Dans ce cadre, de nombreuses activités ont été conduites au CHU du Point $\mathrm{G}$ dont la production locale d'une solution hydro-alcoolique pour la friction des mains, la formation du personnel de soins sur les indications de l'hygiène des mains, l'observation des pratiques d'hygiène des mains, la restitution des résultats d'observance et d'autres outils de promotion (affichage de messages de sensibilisation, aides mémoires) ${ }^{[4,5]}$. Cette intervention pilote a été clôturée en juillet 2008 et a apporté des résultats très encourageants en terme de faisabilité et d'efficacité de la promotion de l'hygiène des mains dans un pays à bas revenu économique. 
L'utilisation des gants médicaux à usage unique a été identifiée comme l'une des principales cibles pour une amélioration future de l'observance de l'hygiène des mains ${ }^{[6,7]}$. En absence d'une intervention ciblée, pendant la campagne d'hygiène des mains au CHU du Point G, la disponibilité des gants de soins s'est considérablement améliorée dans toutes les unités pilotes ${ }^{[4]}$. Cependant, leur usage semble être fréquemment inapproprié pendant les sessions d'observation des pratiques d'hygiène des mains. Il pourrait, de ce fait, induire le non-respect des indications de l'hygiène des mains. L'objectif de notre étude était d'évaluer l'impact de l'usage des gants sur l'observance de l'hygiène des mains afin de promouvoir leur usage approprié au CHU du Point G.

\section{Matériel et méthodes}

Il s'agissait d'une étude d'observation et d'intervention (formation et sensibilisation) réalisée d'avril 2010 à octobre 2011 sur l'usage des gants médicaux au cours des soins. L'étude a été réalisée au CHU du Point G qui est un hôpital universitaire de troisième référence avec 458 lits d'hospitalisation pour 16 services de soins médicaux et trois services techniques. Les enquêtes ont été menées dans les services ayant accueilli la mise en œuvre de la stratégie de l'OMS pour l'amélioration de l'hygiène des mains au CHU du Point G en 2007 qui sont : Chirurgie Générale A, Chirurgie Générale B, Gynécologie Obstétrique, Anesthésie Réanimation, Maladies Infectieuses, Médecine Interne, Néphrologie, Urologie et Urgences. Le recueil du consentement éclairé a été réalisé du 15 avril au 30 mai 2010 et a concerné les neuf services inclus et 274 agents permanents ayant un contact direct avec les patients. L'approche utilisée pour le remplissage des fiches de consentement éclairé était le remplissage par le soignant en présence de l'enquêteur qui pouvait au besoin donner des explications. Les opportunités observées auprès des services de soins étaient au nombre de 400 calculé à partir de la formule de la comparaison de deux échantillons indépendants accessible sur le site http://www.stat.ubc.ca/ rollin/stats/ssize/b2.html ${ }^{[\mathbf{8}]}$. Avec une projection d'amélioration d'observance à l'hygiène des mains obtenue avec l'étude de Allegranzi ${ }^{[4]}$ de $20 \%$ à $30 \%$ après l'intervention, un risque $\boldsymbol{\alpha}$ de $5 \%$ et une puissance de $90 \%$, nous avons obtenus 400 opportunités après majoration. L'opportunité d'hygiène des mains détermine le nombre de fois où l'action d'hygiène des mains est nécessaire. Ainsi plusieurs opportunités peuvent être observées chez un même agent au cours des soins. Les observations avant intervention (phase I) ont été menées du 24 août 2010 au 26 novembre 2010 et du 19 août 2011 au 20 octobre 2011 après intervention (phase II). Ces observations ont été effectuées aux près du personnel ayant donné son consentement. Entre les deux observations, des interventions ont été entreprises à l'endroit du personnel soignant. Ces interventions ont consisté à la formation et à l'affichage de posters sur les cinq indications de l'hygiène des mains (avant de toucher un patient, avant un geste aseptique, après un risque d'exposition à un liquide biologique, après avoir touché un patient, après avoir touché l'environnement d'un patient) et l'indication du port des gants soins médicaux (risque d'exposition aux liquides biologiques). Une équipe de trois observateurs formés sur la méthodologie d'observation de l'OMS ${ }^{[9,10]}$ était chargée de l'observation des pratiques d'hygiène des mains et d'usage des gants médicaux. Un formulaire d'observation adapté à l'usage des gants a été utilisé et comportait la catégorie professionnelle, le service, les indications et les opportunités d'action de l'hygiène des mains et du port de gants. Les observations directes ont été discrètes mais ouvertes, c'est à dire menées auprès de soignants préalablement informés du but et du programme. Ces observations ont été conduites de façon anonyme pendant des sessions de 20 minutes et selon l'approche des «5 Indications» de l'hygiène des mains et l'indication à l'usage des gants médicaux ${ }^{[9,10]}$. Des formulaires d'observation ont été utilisés et les interventions ont concerné toutes les catégories professionnelles, soit un total de 452 soignants recensés dont 274 permanents. Le taux d'observance a été calculé selon la formule suivante: observance $(\%)=$ nombre d'actions réalisées / nombre d'opportunités observées x 100 . Le taux d'usage approprié des gants a été exprimé par la proportion de ports de gants observés en présence d'indications à leur usage. L'impact du port de gants sur la non-observance de l'hygiène des mains, a été mis en évidence par la différence entre les rapports du nombre d'actions d'hygiène des mains non réalisées en présence et en absence du port de gants. Les données ont été saisies et analysées sur le logiciel Epi Info (version 3.4.3) du Center for Disease Control and Prevention, Atlanta, GA, USA. Le test de $\mathrm{Chi}^{2}$ a été utilisé pour comparer les différentes fréquences observées pendant les deux phases. Les valeurs de $p<0,05$ ont été considérées comme statistiquement significatives.

\section{Résultats}

$\mathrm{Au}$ total, 143 fiches de consentement éclairé remplies ont été retournées sur une prévision de 274 fiches soit $52,19 \%$. Cent quatre-vingt-deux sessions d'observations ont été réalisées (80 en phase I et 102 en phase II) au cours desquelles un total de 1185 opportunités d'hygiène des mains a été observé soit une moyenne de 7 opportunités par session. Une session d'observation a duré en moyenne $28,5 \pm 12 \mathrm{mn}$ pour la phase I et $21,4 \pm 9$ $\mathrm{mn}$ lors de la deuxième phase. Les taux 
d'observance globale calculés ont été de 52,05\% pour la phase I et $42,97 \%$ pour la phase II. L'observance a baissé de façon significative à la deuxième période avec $\mathrm{p}=0,0017$.

Durant les deux phases de l'étude, les taux d'observance les plus élevés ont été enregistrés chez les chirurgiens $(80,95 \%$ en phase I et $80,00 \%$ en phase II) et les sages-femmes $(85,71 \%$ en phase I et $80,00 \%$ en phase II). Les faibles taux d'observance à l'hygiène des mains ont été enregistrés chez les aides-soignants soit $28,92 \%$ en phase I et $24,44 \%$ en phase II (tableau I). Le tableau II montre que le personnel était plus observant dans l'indication de l'hygiène des mains «après avoir touché un patient » à la phase $\mathrm{I}(74,58 \%)$ et à la phase II $(66,13 \%)$. Le taux global de port des gants par rapport aux opportunités observées était de $23,30 \%$ durant la première phase de l'étude et de $27,20 \%$ à la deuxième phase (tableau III). Cette différence n'était pas significative avec $p=0,12$. Le taux d'usage approprié des gants a baissé de façon significative $(p<0,0001)$ en passant de $91,50 \%$ durant la phase I à $63,50 \%$ durant la phase II (tableau IV). Le taux d'actions d'hygiène des mains non réalisées avec port de gants était de $31,30 \%$ à la première phase contre $37,50 \%$ à la deuxième phase (tableau V). Le port de gants n'avait aucun impact sur la non-observance à l'hygiène des mains $(p=0,1)$.

\section{Discussions}

L'organisation des soins dans les services ne permettait pas d'observer à la phase II toutes les personnes observées à la phase I et formées sur la pratique de l'hygiène des mains et les indications du port de gant, constituant ainsi une limité de l'étude. L'évaluation de l'usage des gants par rapport à l'hygiène des mains a montré un taux global d'observance à l'hygiène des mains de $52,05 \%$ durant la première phase avec une baisse significative à $42,97 \%$ à la deuxième phase ( $\mathrm{p}=$ $0,0017)$. Nos résultats sont similaires à ceux de l'étude de Rose $\mathrm{L}^{[11]}$ mais différent des études de Ashu $\mathrm{S}^{[\mathbf{1 2}]}$, de Mestre G. ${ }^{[13]}$ et de Sakihama $\mathrm{T}^{[14]}$ qui ont trouvées respectivement un taux d'amélioration de $31,36 \% ; 25 \%$ et $15 \%$. Cette différence avec nos résultats pourraient s'expliquer par l'organisation des services, l'insuffisance dans la disponibilité des gants et des produits pour l'hygiène des mains. Au niveau des catégories professionnelles, pendant les deux phases de l'étude, les infirmiers avaient une observance plus faible (phase I : 50,35\% ; phase II : $39,90 \%$ ) par rapport aux médecins (phase I : $62,15 \%$; phase II : $55,19 \%$ ). Ce qui était conforme aux résultats des études antérieures d'observation réalisées dans les mêmes services ${ }^{[4,5]}$ et celles réalisées au Ghana ${ }^{[15]}$ en 2009 et en Inde ${ }^{[16,17]}$ en 2010 et 2011 avec respectivement $23,1 \%$ (Ghana), $66,1 \%$ et $50,8 \%$ pour les médecins contre $21,1 \%$ (Ghana), $60,7 \%$ et $41,3 \%$ pour les infirmiers. Nos résultats sont contraires à d'autre résultat retrouvé dans la littérature [19-21,] avec des différences variant de $8 \%$ à $29 \%$ en faveur des infirmiers. Par rapport aux indications de l'hygiène des mains, au cours des deux phases d'observation, le taux d'observance de l'hygiène des mains était plus élevé aux indications «Après avoir touché un patient» et «Après un risque d'exposition à un liquide biologique » par rapport aux indications «Avant contact patient» et « Avant acte aseptique ». Cette tendance de l'observance de l'hygiène des mains des soignants est conforme aux résultats des études antérieures d'observation réalisées dans les mêmes services ${ }^{[4,5]}$ et a été rapportée dans plusieurs autres études ${ }^{[19,20,21,22]}$ confirmant ainsi que le soignant a tendance à se protéger d'abord. Le port de gants n'avait aucun impact sur la nonobservance de l'hygiène des mains. Le taux global du port des gants est passé de $23,30 \%$ à la première phase de l'étude à $27,20 \%$ à la deuxième phase. Ces deux valeurs sont inferieures à la fréquence globale du port des gants de $67,8 \%$ rapportée par une étude réalisée en France en 1999 sur le port des gants à usage unique non stériles ${ }^{[23]}$. En considérant la seule indication du port des gants relative au risque d'exposition à un liquide biologique pour apprécier le caractère approprié du port des gants, la majorité des opportunités observées en présence de gants étaient dans un cadre approprié. Cependant, d'autres aspects du port des gants n'étaient pas respectés : une seule paire de gants à usage unique était utilisée pour plusieurs patients et le changement de gants n'était pas aussi respecté lors du passage d'une zone sale à une zone propre chez le même patient.

\section{Conclusion}

Les résultats des deux évaluations avant et après intervention ont montré une amélioration du taux global de port des gants qui n'était pas significative mais sans impact sur l'observance de l'hygiène des mains.

Le taux d'observance de l'hygiène des mains a diminué ce qui nécessiterait l'amélioration dans l'organisation des services, la disponibilité des gants et des produits pour l'hygiène des mains. Les résultats positifs doivent être soutenus et renforcés par une meilleure organisation des formations et sensibilisations en rapport avec l'hygiène des mains et le port approprié des gants.

\section{References}

1- Pittet D, Donaldson L. Clean Care is Safer Care: a worldwide priority. Lancet 2005; 366: 1246-7.

2- World Health Organization. The Global Patient Safety Challenge 2005-2006 "Clean Care is Safer Care". Geneva, WHO, 2005.

3- Allegranzi B, Pittet D. Healthcare-associated infection in developing countries: simple solutions 
to meet complex challenges. Infect Control Hosp Epidemiol 2007; 28: 1323-27

4- Allegranzi B, Sax H, Bengaly L, Richet H, Minta DK, Chraiti MN et al. Successful implementation of the World Health Organization hand hygiene improvement strategy in a referral hospital in Mali, Africa. Infect Control Hosp Epidemiol 2010; 31(2):133-141.

5- Bengaly L. Implantation et évaluation d'un programme de promotion d'hygiène des mains dans un hôpital national du mali Thèse $\mathrm{N}^{\circ} 4318$ Université de Genève 2011. 2011.

6- Zimakoff J, Stormark M, Olesen Larsen S. Use of gloves and handwashing behaviour among health care workers in intensive care units. A multicentre investigation in four hospitals in Denmark and Norway. Journal of Hospital Infection 1993;24:6367 ;

7- Basurrah MM, Madani TA. Handwashing and gloving practice among health care workers in medical and surgical wards in a tertiary care centre in Riyadh, Saudi Arabia. Scand J Infect Dis 2006;38(8):620-4.

8- Brant R. Inference for Proportions: Comparing Two Independent Samples disponible sur http://www.stat.ubc.ca/ rollin/stats/ssize/b2.html . Consulté le 25 juillet 2015.

9- Sax H, Allegranzi B, Chraiti MN, Boyce J, Larson E, Pittet D. The World Health Organization hand hygiene observation method. Am J Infect Control 2009; 37(10):827-834.

10- WHO. Hygiène des Mains : Manuel Technique de Référence à l'attention des professionnels soignants, des formateurs et des observateurs des pratiques d'hygiène des mains; Organisation Mondiale de la Santé 2010; disponible sur http://www.who.int/gpsc/5may/tools/training_ed ucation/gpsc hhtool TRM 2010 40 fr.pdf. Consulté le 25 juillet 2015.

11- Rose L, Rogel K, Redl L, Cade JF. Implementation of a multimodal infection control program during an Acinetobacter outbreak. Intensive Crit Care Nurs 2009; 25(2):57-63.

12- Ashu S. M, Smitha E. G, John A. Efficacy of a multimodal intervention strategy in improving hand hygiene compliance in a tertiary level intensive care unit. Indian J Crit Care Med. 2011 Jan-Mar; 15(1): 6-15.

13- Mestre G, Berbel C, Tortajada P, Alarcia M, Coca R, et al. (2012) "The 3/3 Strategy": A Successful Multifaceted Hospital Wide Hand Hygiene
Intervention Based on WHO and Continuous Quality Improvement Methodology. PLoS ONE 7(10): e47200. October 2012

14- Sakihama $\mathrm{T}$, Honda $\mathrm{H}$, Saint $\mathrm{S}$ et al. Improving healthcare worker hand hygiene adherence before patient contact: A multimodal intervention of hand hygiene practice in Three Japanese tertiary care centers. J Hosp Med. 2015 Oct 1. doi: 10.1002/jhm.2491.

15- Asare A, Enweronu-Laryea CC, Newman MJ. Hand hygiene practices in a neonatal intensive care unit in Ghana. J Infect Dev Ctries 2009;3:352-356.

16- Sahay S, Panja S, Ray S, Rao BK. Diurnal variation in hand hygiene compliance in a tertiary level multidisciplinary intensive care unit. Am J Infect Control 2010;38:535-539.

17- Sharma S, Sharma S, Puri S, Whig J. Hand hygiene compliance in the intensive care units of a tertiary care hospital. Indian J Community Med. 2011 JulSep;36(3):217-21. doi: 10.4103/0970-0218.86524

18- Sakihama T, Honda H., Saint S, Fowler K.E, Shimizu T et Al. Hand Hygiene Adherence Among Health Care Workers at Japanese Hospitals: A Multicenter Observational Study in Japan. J Patient Saf . 2014;00: 00-00)

19- Pittet D, Mourouga P, Perneger TV. Compliance with handwashing in a teaching hospital. Infection Control Program. Ann Intern Med 1999; 130(2):126-130.

20- Pittet D, Hugonnet S, Harbarth S, Mourouga P, Sauvan V, Touveneau $S$ et al. Effectiveness of a hospital-wide programme to improve compliance with hand hygiene. Infection Control Programme. Lancet 2000; 356(9238):1307-1312.

21- Erasmus V, Daha TJ, Brug H, Richardus JH, Behrendt MD, Vos MC et al. Systematic review of studies on compliance with hand hygiene guidelines in hospital care. Infect Control Hosp Epidemiol 2010; 31(3):283-294.

22- Saba R, Inan D, Seyman D, Gul G, Senol YY, Turhan $\mathrm{O}$ et al. Hand hygiene compliance in a hematology unit. Acta Haematol 2005; 113(3):190193.

23- CCLIN Sud-Ouest. AUDIT SUR LES PRATIQUES: Le port des gants à usage unique non stériles 1999. disponible sur http://www.cclinsudouest.com/wpcontent/uploads/2015/05/gants9 9.pdf . Consulté le 25 juillet 2015 
Tableau I : Répartition de l'observance de l'hygiène des mains selon la catégorie professionnelle durant les deux phases

\begin{tabular}{|c|c|c|c|c|c|c|c|}
\hline \multirow[b]{2}{*}{ Catégories Professionnelles } & \multicolumn{3}{|c|}{ Phase I } & \multicolumn{3}{|c|}{ Phase II } & \multirow[b]{2}{*}{$\mathrm{p}$} \\
\hline & Actions & Opportunités & $\begin{array}{c}\text { Observance } \\
(\%)\end{array}$ & Actions & Opportunités & $\begin{array}{c}\text { Observance } \\
(\%)\end{array}$ & \\
\hline Aides-soignantes & 24 & 83 & 28,92 & 22 & 90 & 24,44 & 0,62 \\
\hline Anesthésistes/Réanimateurs & 7 & 14 & 50,00 & 8 & 18 & 44,44 & 0,96 \\
\hline Chirurgiens & 68 & 84 & 80,95 & 56 & 70 & 80,00 & 0,95 \\
\hline Élèves infirmiers & 19 & 30 & 63,33 & 2 & 14 & 14,29 & 0,006 \\
\hline Étudiants en médecine & 31 & 75 & 41,33 & 27 & 87 & 31,03 & 0,23 \\
\hline Infirmiers & 72 & 143 & 50,35 & 77 & 193 & 39,90 & 0,05 \\
\hline Médecins & 58 & 116 & 50,00 & 53 & 124 & 42,74 & 0,26 \\
\hline Sages-femmes & 12 & 14 & 85,71 & 24 & 30 & 80,00 & 1 \\
\hline TOTAL & 291 & 559 & 52,05 & 269 & 626 & 42,97 & $\mathbf{0 , 0 0 1 7}$ \\
\hline
\end{tabular}

Tableau II : Répartition de l'observance de l'hygiène des mains selon les indications pendant les deux phases de l'étude

\begin{tabular}{|c|c|c|c|c|c|c|}
\hline \multirow{2}{*}{$\begin{array}{c}\text { Indications de l'hygiène des } \\
\text { mains }\end{array}$} & \multicolumn{3}{|c|}{ Phase I } & \multicolumn{3}{|c|}{ Phase II } \\
\hline & $\begin{array}{c}\text { Action } \\
\mathrm{s}\end{array}$ & Opportunités & $\begin{array}{c}\text { Observance } \\
(\%)\end{array}$ & Actions & Opportunités & $\begin{array}{c}\text { Observance } \\
(\%)\end{array}$ \\
\hline Avant de Toucher un patient & 65 & 119 & 54,62 & 82 & 239 & 34,31 \\
\hline Avant un Geste Aseptique & 50 & 137 & 36,50 & 42 & 154 & 27,27 \\
\hline $\begin{array}{l}\text { Après un Risque d'Exposition } \\
\text { à un Liquide Biologique }\end{array}$ & 74 & 139 & 53,24 & 77 & 121 & 63,64 \\
\hline Après avoir Touché un Patient & 88 & 118 & 74,58 & 164 & 248 & 66,13 \\
\hline $\begin{array}{l}\text { Après avoir Touché } \\
\text { l'Environnement d'un Patient }\end{array}$ & 15 & 50 & 30,00 & 3 & 60 & 5,00 \\
\hline
\end{tabular}

Tableau III : Taux du port des gants suivant les opportunités durant les deux phases de l'étude

\begin{tabular}{|c|c|c|c|c|}
\hline \multirow[b]{2}{*}{ Port de gants } & \multicolumn{2}{|c|}{ Phase I } & \multicolumn{2}{|c|}{ Phase II } \\
\hline & Opportunité & $\%$ port de Gant & Opportunité & $\%$ port de gant \\
\hline Oui & 130 & 23,30 & 170 & 27,20 \\
\hline Non & 429 & 76,70 & 456 & 72,80 \\
\hline Total & 559 & 100 & 626 & 100 \\
\hline
\end{tabular}


Tableau IV: Taux d'usage approprié des gants suivant les indications recommandées pendant les deux phases de l'étude

\begin{tabular}{lccccc}
\hline & \multicolumn{2}{c|}{ Phase I } & \multicolumn{2}{c}{ Phase II } \\
\cline { 2 - 5 } Indication Port des gants & Opportunités & $\begin{array}{c}\text { \% port } \\
\text { de gant }\end{array}$ & & Actions & Opportunités \\
\hline Oui & 119 & $\mathbf{9 1 , 5 0}$ & 108 & $\mathbf{6 3 , 5 0}$ \\
Non & 11 & 8,50 & 62 & 36,50 \\
\hline & Total & 130 & 100 & 170 & 100 \\
\hline
\end{tabular}

Tableau V : Répartition des actions d'hygiène des mains non réalisées suivant les deux phases de l'évaluation

\begin{tabular}{lccccc}
\hline \multirow{2}{*}{$\begin{array}{c}\text { Actions d'hygiène des mains } \\
\text { non réalisées }\end{array}$} & \multicolumn{2}{c|}{ Phase I } & \multicolumn{2}{c}{ Phase II } \\
\cline { 2 - 6 } & Nombre & $\%$ & Nombre & $\%$ \\
\hline Avec port de gants & 84 & 31,30 & 134 & 37,50 \\
Sans port de gants & 184 & 68,70 & 223 & 62,50 \\
\hline & Total & 268 & 100 & $\mathbf{3 5 7}$ & 100 \\
\hline
\end{tabular}

